\title{
Toe Graphaesthesia Deficits Following TBI: An Indicator of Dysfunction within the Medial Prefrontal Surface of the Human Cerebrum
}

Paula L Corradini and Michael A Persinger*

Departments of Psychology and Biology, Laurentian University, Sudbury, Ontario, Canada

\begin{abstract}
Background: Discerning dysfunction or damage by classic neuropsychological tests within the anterior medial surfaces of the cerebrum and the tissue influenced by the distributions of the Anterior Cerebral Artery following Traumatic Brain Injuries (TBI) has been ambiguous. Toe graphaesthesia (TG) has been shown to be associated with increased perfusion rates as measured by Single Positron Emission Computerized Tomography (SPECT) within the medial prefrontal region. The utility of TG to discern impairment was investigated.
\end{abstract}

Methods: A total of 25 patients who has sustained TBI secondary to motor vehicle incidents and who were referred for a full neuropsychological assessment to discern level of function were administered the TG while 19 quantitative electroencephalographic (QEEG) measurements were recorded.

Results: Toe graphaesthesia scores were significantly impaired ( $z$ scores>-2.0) for patients whose Neuropsychological Impairment Indices were greater than 0.3 for the Halstead-Reitan Index. The only QEEG variable that correlated with the TG error rate was lower power for high beta activity over the central channels.

Conclusions: Standardized scores for toe graphaesthesia, which requires about ten minutes to administer, is a valid indicator of the functional integrity of the medial prefrontal region and has now been validated by QEEG and SPECT measurements.

Keywords: Toe graphaesthesia; TBI; QEEG; Middle prefrontal cortices; Differential diagnosis

\section{Introduction}

The convergence of decades of experimental research involved with focal vs. diffuse brain lesions and clinical inferences from primarily severe, battlefield acquired cerebral injuries (Traumatic Brain Injuries, TBI) was manifested in the Halstead-Reitan Index for Neuropsychological Impairment [1]. From a variety of different psychometric indicators that were available during the first half of the twentieth century cut-off scores from seven different measures were employed to discern the threshold for neuropsychological impairment and implicitly brain injury. Five tests were finally selected: finger agility (Finger Tap), Seashore Rhythm, Speech-Sounds, The Category Test, and the Tactual Performance Test (TPT). Both traditional and contemporary imaging studies indicate that these tests reflect the general activity of the frontal, temporal, and parietal lobes of the lateral surface of the cerebrum. Even a novel abbreviated index [2] derived from the three standardized test scores that maximally discriminated patients who displayed brain injury vs. those who did not according to traditional criteria involved temporal lobe (dichotic word listening) frontal lobe (Trails A) and parietal lobe (TPT) tasks.

However, the functional integrity of the medial surface of the cerebral hemispheres is not represented in traditional indicators of neuropsychological impairment. The anterior two-thirds of this region are supplied by the Anterior Cerebral Artery (ACA) whereas the lateral surface is primarily supplied by the Middle Cerebral Artery. The ACA in the general population displays a remarkably varied angioarchitecture and pattern of distribution. Approximately $20 \%$ of the population has only one original branch (in one hemisphere) that bifurcates and crosses to the contralateral hemisphere [3]. Consequently these individuals would be more prone to torsional stresses from differential acceleration of mechanical pressures propagating through and between the hemispheres that could disrupt vascular supply to this region. These stresses could arise from concussive forces or the asymmetrical propagation of mechanical energies through the cerebral volume which could result in brief ischemia and potentially permanent compromise of neuronal function.

Chronic dysfunction in this region could follow brief ischemias and would not necessarily be reflected in traditional neuropsychological measures. Classical neurofunction data indicated that tactile information for the toes is represented along the medial, paracentral parietal lobe; this region for toe gnosis discrimination has recently been verified by in our laboratory by s_LORETA (Low Resolution Electromagnetic Tomography). Toe Graphaesthesia (TG) which involves printing numbers with a stylus on the bottom of the toes, using the same format as for Finger Graphaesthesia, was shown by SPECT more than a decade ago to be associated with increased uptake of tracer along the medial prefrontal surface [4]. As predicted from principles of neuroscience, the standardized scores for TG are correlated with those for foot tap [5]. Unlike many other single test measures TG scores were significantly correlated with a variety of other scores including Design Fluency, Auditory Closure, Foot Agility, TPT (the dominant hand, both hands, and memory score), dichotic word listening (both ears) and the Halstead Reitan Impairment Index, HRII [5].

Over the last 20 years we have found that patients referred for

*Corresponding author: Clinical Neuroscience Laboratory, Behavioura Neuroscience Program, Departments of Psychology and Biology, Laurentian University, Sudbury, Ontario, Canada P3E 2C6, Tel: 01-705-675-4824; Fax: 01705-671-3844; E-mail: mpersinger@laurentian.ca; pl_corradini@laurentian.ca

Received October 31, 2013; Accepted November 26, 2013; Published December 04, 2013

Citation: Corradini PL, Persinger MA (2013) Toe Graphaesthesia Deficits Following TBI: An Indicator of Dysfunction within the Medial Prefrontal Surface of the Human Cerebrum. J Neurol Neurophysiol 4: 177. doi:10.4172/2155-9562.1000177

Copyright: (c) 2013 Corradini PL, et al. This is an open-access article distributed under the terms of the Creative Commons Attribution License, which permits unrestricted use, distribution, and reproduction in any medium, provided the original author and source are credited. 
assessment subsequent to TBIs also displayed clinical evidence of ACArelated injuries, as inferred by impaired TG. These scores were impaired $(\mathrm{z}>-3.00)$ while the HRII indices were not $(<0.4)$. The patients who displayed deficits for TG but normal range HRII scores were also those who displayed more clinical depression as inferred by the Depression Scale of the MMPI (Minnesota Multiphasic Personality Inventory). Considering: (1) the convergence of passage of the nonmyelinated, biogenic amine pathways for norepinephrine and serotonin through a region that involves the anterior medial surface, and, (2) hypofunctions in these pathways are one of the strongest correlates of clinical depression, these complications would be expected.

In other words, the z-score for TG is singularly as effective for discerning general neuropsychological impairment as the HRII. Consequently we tested the construct validity of TG measure by examining three groups of increasing degrees of neuropsychological impairment that would constitute the general domain of "mild" to "moderate" TBI, to discern if there was a systematic change in electroencephalographic frequency or power within the areas most proximal to the medial surface. If the effect was as powerful as suspected, we expected the largest differences between levels of impairment groups would involve the central sensor placements most proximal to the frontal medial surface.

\section{Method}

\section{Participants}

Data obtained from TBI patients $(n=22)$ who had undergone full neuropsychological assessments over and approximately three year period (January 2010-April 2013) were analyzed. The mean age of the patients was 38.5 years ( $\mathrm{SD}=14.7$ years). The mean and standard deviations for the delays between the mechanical impacts to the skulls and the assessments were 5.6 and 5 years, respectively (range 0.3 to 16 years). All of them had been referred by external agencies for a full neuropsychological assessment to discern the level of functioning following a closed head injury due to an impact of concussive force or mechanical energies. They all met the criteria of at least a mild TBI on the bases of either a GSC of $>13$ or a suspension of consciousness of less than $20 \mathrm{~min}$.

The designation of 'mild TBI' is of importance because the outcome and quality of life of patients who have sustained these injuries has not been widely studied. Individuals who have sustained mild TBIs tend to greatly vary with respect to their quality of life and neuropsychological impairment levels. Although the outcome for these individuals is favorable, the patients in this study were referred for an assessment because many of them were still experiencing symptoms or were having difficulty adapting post-injury. These patients were being provided treatment from external agencies on an individual basis. For the most part, because these patients met the criteria of a mild TBI treatment was focused on symptoms management. Table 1 shows the information about each patient (e.g. age, sex, time since injury). The overall Halstead-Reitan Impairment Index is a reflection of each patient's neurocognitive functioning at the time of the assessment. In addition, the results from the Toe Graphaesthesia test are shown (z-score and total number of errors).

All patients were administered our standard battery of intellectual, memory, academic achievement, classic (Halstead-Reitan Battery) and novel (e.g. Conditioned Spatial Association Test) neuropsychological and personality tests [6]. Quantitative electroencephalographic measurements were also completed employing a standardized protocol. On the bases of the Russell et al [7] ordinal range (ROR), subjects were assigned to one of three groups: no impairment ( 0 or 1$)$, mild impairment (2) and moderate to severe impairment (3-5). They were partitions of the original Halstead-Reitan Impairment Index between 0 and 1 such that 0 to $0.1=0,0.2$ to $0.3=1,0.4$ to $0.5=2,0.6$ to $0.7=3$, 0.8 to $0.9=4$, and $1.0=5$. The HRII derived from seven scores from the battery. There were 9,7 , and 6 patients in each group (ROR=0-1, 2, 3+) respectively.

\section{Procedure}

Each assessment required two full days and consisted of a battery of traditional and contemporary neuropsychological tests in addition to intellectual, academic achievement, memory, and personality tests. Testing occurred between $10 \mathrm{hr}$ and $18 \mathrm{hr}$. The QEEG data was collected in the final hour of the first day and required $45 \mathrm{~min}$. Responses to the TG test measures the individual's ability to discern numbers written on the distal tips of the toes with a stylus (and the individual did not have the benefit of visual feedback). The total errors for the identification of the toes for both feet were determined. Total errors for the detection of the randomly presented numbers $3,4,5$, and 6 for the toes of both feet (potential error range 0 to 40) were obtained. Table 2 shows the overall Toe Graphaesthesia results for impairment groups (ROR).

\section{Results}

Analysis of variance demonstrated a significant difference between the three groups of patients designated according to the ROR for Toe Graphaesthesia $\left[\mathrm{F}_{(2,21)}=5.00, \mathrm{p}=.018\right.$, eta $\left.{ }^{2}=0.34\right]$. Post hoc analysis indicated that those with mild or moderate-severe impairment according to the ROR displayed significantly more errors for TG

\begin{tabular}{|c|c|c|c|c|c|c|}
\hline $\begin{array}{c}\text { Patient } \\
\#\end{array}$ & Age & Sex & $\begin{array}{c}\text { Time Since } \\
\text { Injury (years) }\end{array}$ & $\begin{array}{c}\text { Halstead } \\
\text { Impairment Index }\end{array}$ & $\begin{array}{c}\text { TG } \\
\text { (z-score) }\end{array}$ & $\begin{array}{l}\text { TG total } \\
\text { errors }\end{array}$ \\
\hline 1 & 15 & Male & 10 & 0.28 & -0.5 & 9 \\
\hline 2 & 18 & Male & 0.3 & 0.28 & -1.2 & 12 \\
\hline 3 & 20 & Male & 15 & 0.43 & -3.6 & 22 \\
\hline 4 & 20 & Male & 0.7 & 0.14 & -0.7 & 10 \\
\hline 5 & 22 & Female & 1 & 0.14 & 1.0 & 3 \\
\hline 6 & 26 & Female & 3 & 0 & -0.2 & 8 \\
\hline 7 & 27 & Female & 6 & 0.86 & -1.9 & 15 \\
\hline 8 & 30 & Female & 6 & 0.43 & -0.2 & 8 \\
\hline 9 & 32 & Female & 16 & 0.14 & 0.2 & 6 \\
\hline 10 & 36 & Female & 2.5 & 0.43 & -0.7 & 10 \\
\hline 11 & 38 & Female & 7 & 0.28 & -1.0 & 11 \\
\hline 12 & 40 & Female & 5 & 0.43 & -0.7 & 10 \\
\hline 13 & 46 & Male & 15 & 0.43 & -4.6 & 26 \\
\hline 14 & 46 & Female & 2 & 0.71 & -4.1 & 24 \\
\hline 15 & 47 & Male & 12 & 0.43 & -3.9 & 23 \\
\hline 16 & 49 & Female & 4 & 0.71 & -2.7 & 18 \\
\hline 17 & 50 & Male & 5 & 0.14 & -0.5 & 9 \\
\hline 18 & 50 & Female & 4 & 1 & 0 & 7 \\
\hline 19 & 54 & Female & 0.5 & 0.71 & -1.9 & 15 \\
\hline 20 & 57 & Male & 0.7 & 0 & -0.7 & 10 \\
\hline 21 & 61 & Male & 5 & 0.57 & -3.4 & 21 \\
\hline 22 & 62 & Male & 1.8 & 0.43 & -1.2 & 12 \\
\hline
\end{tabular}

\begin{tabular}{|c|c|c|}
\hline Impairment level & Mean TG z-score & Average \# of errors \\
\hline No Impairment & -0.4 & 8.7 \\
\hline Mild Impairment & -2.1 & 15.9 \\
\hline Moderate to Severe Impairment & -2.3 & 16.7 \\
\hline
\end{tabular}

Table 2: Toe Graphaesthesia results (overall mean z-score and average number of errors) by impairment level (ROR). 
compared to the group that displayed no ROR impairment. The results are shown in Figure 1. The Kruskall-Wallis test, completed because of the discrepancy in variances, reiterated the statistically significant group differences $\left(\chi^{2}=6.74, \mathrm{p}=.034\right)$.

The results of the correlations between errors for the TG test and spectral power for each of the 7 frequency bands for each of the 19 channels were very specific. The only significant correlation found was with the sum of the power over the central channels in the high beta frequency band ( $\mathrm{rho}=-.46, \mathrm{p}=.03 ; \mathrm{r}=-.57, \mathrm{p}=.006$ ). This result indicated that as proportion of power within the high beta band decreased over the central regions the errors (the greater the deficit) on the TG test increased. These measurements localized the activation for this task over the correct region of the brain, thus providing additional construct validity for the TG test. They have also demonstrated a link between the significance of the overall impairment and this specific task (Figure 1).

\section{Discussion}

The importance of direct patient-to-diagnostician interaction is a central feature of clinical assessment. Although the future of assessment and the development of strategies for intervention and care will very likely be relegated to the results from imagining technology, the utility of a small number of performance-based, standardized measurements that will allow an estimate of level of function of the patient as well as the general region of the likely dysfunction subsequent to the TBI would be beneficial for the patient and the practice. Like the difficulties involved with the "silent" zones in historical electroencephalography when the transient or localized activities within the ventral surface of the cerebrum adjacent the cranium (such as the ventral temporal lobes) were not easily discernable by surface EEG, the dysfunction within the medial surface of the human cerebrum has been more difficult to infer by typical neuropsychological testing. The strength of the effect for TG to discern neuropsychological impairment and the validity of this measure with respect to the general region this measure reflects may help compensate for this disparity of coverage.
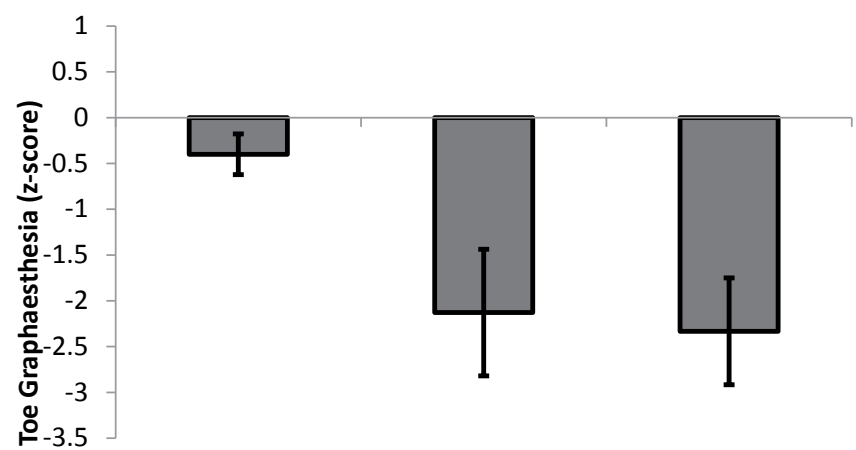

$0-1$

2

Russell Rating Equivalent

Figure 1: Standardized deficit ( $z$ ) scores for Toe Graphaesthesia as a function of the three groups of patients classified according to the Russell criteria as no impairment (0-1), mild impairment (2) and moderate to severe impairment (3+). The no impairment Russell criteria is equivalent to Halstead-Reitan Impairment of $<0.4$.
The ACA and its distributions display remarkable individual differences. The fact that almost a fifth of the brains of the population contain only one rather than two source ACAs and the former bifurcates and traverses the longitudinal fissure to produce the second major branch in the other hemisphere increases this subset of the population to be particularly vulnerable to torque-like mechanical impacts that transiently displace or distort the normal apposition of the two surfaces of the hemispheres. Consequently to the "same" magnitude and directional forces that would be inconsequential to many people could produce pervasive permanent "punctate-like" damage within the cerebral structures that are supplied by the various branches of the anomalous source branch of the ACA in others. Because a major component of the ascending non-myelinated fibers of the norepinephrine and serotonin systems converge and traverse the anterior medial surface before they retroflex and distribute through the upper layers of the cerebral cortices, dysfunction in very focal regions of the anterior medial prefrontal surface would also produce the classic "pseudodepression" which could complicate both diagnosis and treatment [8].

\section{References}

1. Reitan RM, Wolfson D (1985) The Halstead-Reitan Neuropsychological Test Battery. Neuropsychology Press, Tucson, Arizona, USA.

2. Persinger MA (2003) A brief (one hour) quantitative neuropsychological assessment with three performance-based tests: strong concordance with proficiency scores for a more extensive battery. Percep Mot Skil 96: 1005-1012.

3. Burst JCM (1992) Anterior Cerebral Artery disease. In: Barnett HJM, Mohr JP, Stein MS, Yatsu FM (eds.) Stroke: Pathophysiology, diagnosis and management. Churchill Livingstone: NY, USA, 337-360.

4. Persinger MA, Webster D, Tiller SG (1998) SPECT (HMPAO) support fo activation of the medial prefrontal cortices during toe graphaesthesia. Percept Mot Skills 87: 59-63.

5. Persinger MA, Richards PM (1995) Foot agility and toe gnosis/graphaesthesia as potential indicators of integrity of the medial cerebral surface: normative data and comparison with clinical populations. Percept Mot Skills 80: 1011-1024.

6. Gorham R, Persinger MA (2012) Emergence of complex partial epilepsylike experiences following closed head injuries: personality variables and neuropsychological profiles. Epilepsy Behav 23: 152-158.

7. Russell EW, Neuringer C, Goldstein G (1970) A multiple scoring method fo the assessment of complex motor functions. J Con Clin Psychol 43:800-809.

8. Persinger MA (1997) Depression following brain trauma is enhanced in patients with mild discrepancies between intelligence and impairment on neuropsychological scores. Percept Mot Skills 84: 1284-1286. 